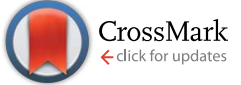

Cite this: Energy Environ. Sci., 2014, 7, 3424

\section{Measurement of minority-carrier diffusion lengths using wedge-shaped semiconductor photoelectrodes $\uparrow$}

\author{
Ragip A. Pala, $\$$ Andrew J. Leenheer, $\$$ Michael Lichterman, Harry A. Atwater* \\ and Nathan S. Lewis*
}

Measurement of the photocurrent as a function of the thickness of a light absorber has been shown herein both theoretically and experimentally to provide a method for determination of the minority-carrier diffusion length of a sample. To perform the measurement, an illuminated spot of photons with an energy well above the band gap of the material was scanned along the thickness gradient of a wedgeshaped, rear-illuminated semiconducting light absorber. Photogenerated majority carriers were collected through a back-side transparent ohmic contact, and a front-side liquid or Schottky junction collected the photogenerated minority carriers. Calculations showed that the diffusion length could be evaluated from the exponential variation in photocurrent as a function of the thickness of the sample. Good agreement was observed between experiment and theory for a solid-state silicon Schottky junction measured using this method. As an example for the application of the technique to semiconductor/ liquid-junction photoelectrodes, the minority-carrier diffusion length was determined for graded thickness, sputtered tungsten trioxide and polished bismuth vanadate films under back-illumination in contact with an aqueous electrolyte. This wedge technique does not require knowledge of the spectral absorption coefficient, doping, or surface recombination velocity of the sample.
Received 22nd May 2014

Accepted 29th July 2014

DOI: $10.1039 / \mathrm{c} 4 \mathrm{ee} 01580 \mathrm{k}$

www.rsc.org/ees

\begin{abstract}
Broader context
The development of solar fuel systems that convert clean and abundant solar energy to efficient fuels can provide a carbon-neutral energy resource for the future. An efficient solar-fuel system requires light absorbing materials with high quantum efficiency and a band gap that matches the free energy of the water-splitting reaction. However there are few Earth-abundant semiconducting light absorbers that meet these requirements and have a sufficiently long minority-carrier diffusion length to allow charge-carrier collection. To identify and develop new materials, rapid characterization techniques are required to evaluate their electrical and optical properties. Here we theoretically and experimentally demonstrate a simple technique for minority-carrier diffusion length measurement in semiconductors. Our technique utilizes the measurement of photocurrent decay along the thickness gradient of a wedge-shaped semiconductor film and does not require knowledge of the surface recombination velocity or spectral absorption coefficient of the sample. The proposed technique is quite general and applicable for wide range of semiconductor systems.
\end{abstract}

\section{Introduction}

New light absorbers are of interest for both photovoltaic and photoelectrochemical cell (PEC) applications. ${ }^{1}$ Specifically for PEC applications, the required materials must be stable and must provide, either alone or in combination, the photovoltage

Joint Center for Artificial Photosynthesis, California Institute of Technology, 100 Jorgensen Laboratory, MC 139-74, Pasadena, California, 91125, USA. E-mail: haa@ caltech.edu; nslewis@caltech.edu

$\dagger$ Electronic supplementary information (ESI) available: Additional equations for photocurrent calculations, $\mathrm{XRD}$ for $\mathrm{WO}_{3}$ deposited at various temperature, optical determination of thickness profile, cyclic voltammetry scan of $\mathrm{WO}_{3}$ electrodes, and further details on the ALD processing and polishing. See DOI: 10.1039/c4ee01580k

$\$$ Authors with equal contribution. needed to drive the fuel-forming reactions of concern, such as water splitting and/or reduction of $\mathrm{CO}_{2}$. Automated methods of producing new semiconducting materials ${ }^{2-5}$ have resulted in a growing number of materials that appear to be promising candidates for use in PEC systems. ${ }^{6}$ Properties such as the band gap and carrier lifetimes ${ }^{7,8}$ can be screened rapidly, but a critical property for the realization of a high performance PEC cell, the minority-carrier diffusion length, $L$, requires detailed measurement techniques and protocols. The minority-carrier diffusion length is the average distance that a minority carrier can travel before recombining, and is proportional to the square root of the minority-carrier lifetime.

Electron beam-induced current ${ }^{9}$ (EBIC) and spectral response ${ }^{10}$ techniques are widely used to obtain the value of $L$ in semiconductors. EBIC yields a value of $L$ even when $L$ is short $(<0.1 \mu \mathrm{m}) .{ }^{11}$ In one approach, the sample is configured so that a 
solid-state junction lies parallel to the sample surface. The energy of an electron beam is varied while the beam is incident upon the junction, and $L$ can then be derived from measurements of the induced current. Alternatively, the sample can be configured so that the solid-state junction is oriented normal to the sample surface, with $L$ derived from the current produced as the electron beam is scanned along the surface of the sample. ${ }^{12}$ When the junctions are oriented parallel to the surface, the data analysis can be quite complex. When the junctions are normal to the surface, surface recombination as well as the difficulty of preparing a perpendicular junction complicate the implementation of EBIC to measure $L \cdot{ }^{13}$ In the spectral response technique, $L$ is obtained by measurement of either the photocurrent or photovoltage as a function of the wavelength of light incident on a thick sample (film thickness $\gg L$ ). However, determination of $L$ from spectral response data requires accurate values of the absorption length near the band gap of the material. ${ }^{14}$ Techniques based on scanning light illumination, ${ }^{15,16}$ e.g. light beam-induced current (LBIC), can provide the minority-carrier diffusion lengths simply without a need for optical characterization of the material, though they require diffusion lengths that are orders of magnitude higher than the illumination wavelength.

We describe herein a technique that allows measurement of the minority-carrier diffusion length of a material by scanning strongly absorbed illumination along the back side of a photovoltaic or PEC device that has been prepared with a gradient in the thickness of the light-absorbing layer (see

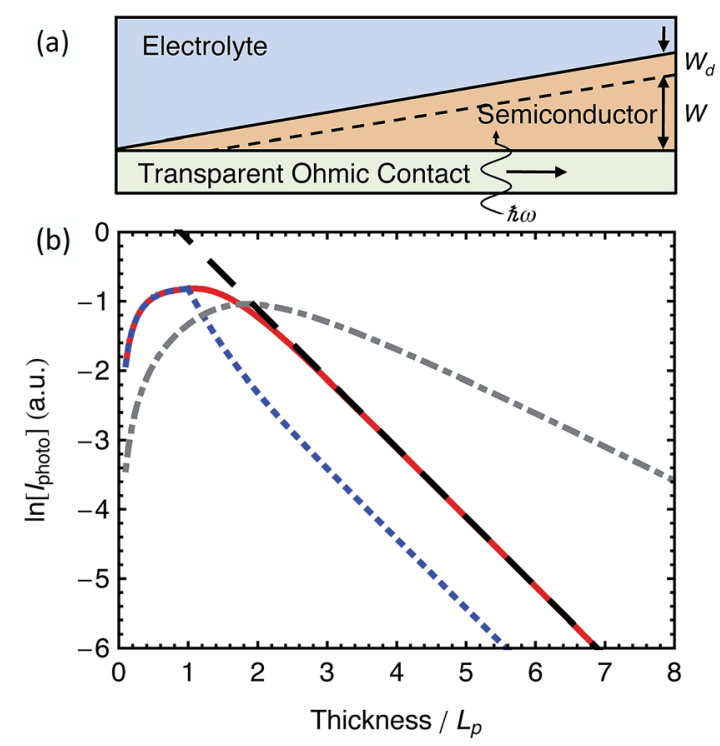

Fig. 1 (a) Schematic of the measurement geometry. (b) Example of calculated photocurrent as a function of thickness, for strongly absorbed light. The solid red curve illustrates a case with a low rate of recombination at the back surface and $L_{p}=4 / \alpha$. The dotted blue curve illustrates a case with a high rate of recombination at the back surface. The dashed black curve is the slope corresponding to the rate of exponential decay of $J_{\text {photo. }}$. The dot-dashed grey curve illustrates the regime where the light is weakly absorbed $\left(L_{p}=0.5 / \alpha\right)$ and $L_{p}$ cannot be extracted from the data. In all cases we assumed $W_{d}=L_{p}$.
Fig. 1a). Though denoted previously for polished solid-state junctions as the "angle-lapped junction" technique, ${ }^{10}$ we demonstrate the applicability of the technique to liquid junctions and wedge-shaped absorbers.

\section{Theory}

An abrupt junction, low-level injection, and full collection of minority carriers at the depletion region boundary were assumed for all samples. Under such conditions, for a specific thickness of the light-absorbing layer, the photocurrent density $(J)$ can be calculated from the one-dimensional diffusion equation and the current continuity equation, including variable surface recombination rates at the illuminated back side of the device. The details of the calculation can be found in the literature ${ }^{10}$ as well as in the ESI. $\dagger$

For an n-type semiconductor with a hole diffusion length, $L_{\mathrm{p}}$, in the limit of strongly absorbed illumination incident upon the back side of a device with a quasineutral region thickness $W$ a few times greater than the diffusion length, the photocurrent reduces to:

$$
\left|J_{\text {photo }} / e_{\mathrm{c}}\right| \approx 2(1-R) \Phi\left(1+\frac{S}{\alpha L_{\mathrm{p}}}\right)(1+S)^{-1} \mathrm{e}^{-\frac{W}{L_{\mathrm{p}}}}
$$

where $e_{\mathrm{c}}$ is the charge on an electron, $R$ is the reflectivity of the surface, $\Phi$ is the incident light flux, $\alpha$ is the absorption coefficient and $S$ is a dimensionless parameter equal to $s L_{\mathrm{p}} / D_{\mathrm{p}}$ that describes the surface recombination velocity $(s)$ at the back side of the device relative to the minority-carrier diffusion coefficient $\left(D_{\mathrm{p}}\right)$. Because the pre-exponential terms do not depend upon $W$, the minority-carrier diffusion length, $L_{\mathrm{p}}$, can be extracted from the slope of a plot of $\ln \left[J_{\text {photo }}\right] v s$. $W$. Eqn (1) indicates that a high rate of surface electron-hole recombination at the back side of the device will reduce the magnitude of the photocurrent but will not affect the rate of the exponential decay of $J$ as a function of the thickness of the sample. Note that analysis of the photocurrent under back illumination does not require knowledge of the depletion width, but the sample must be thick enough to support a quasineutral region.

In the opposing limit of weakly absorbed light for which $1 / \alpha$ $\gg W$ the photocurrent reduces to the form.

$$
\left|J_{\text {photo }} / e_{\mathrm{c}}\right| \approx \frac{(1-R) \Phi \tau \alpha}{\left(1+\alpha L_{\mathrm{p}}\right) L_{\mathrm{p}}} \mathrm{e}^{-\alpha W}
$$

In this situation, the rate of the exponential decay of $J$ as a function of $W$ is determined by the absorption length, $1 / \alpha$, rather than by the minority-carrier diffusion length, $L_{\mathrm{p}}$. A numerical analysis of the full photocurrent equation indicated that an accurate value of $L_{\mathrm{p}}$ could be determined from the slope of a plot of $J v s . W$, provided that $L_{\mathrm{p}}>1 / \alpha$. The absorption length of the light used in the measurement therefore provides a lower limit on the diffusion length that can be measured using this technique.

Fig. 1b depicts the behaviour of the photocurrent as a function of thickness, for three different limits of the surface recombination rate and absorption length. The $x$-axis is a 
dimensionless thickness such that the exponential decay of eqn (1) is evident as a slope of -1 in the plot. The red solid line depicts a base case for strongly absorbed light and conditions that result in negligible recombination of carriers at the back surface, e.g. a well-passivated semiconductor surface. The photocurrent increases with thickness until the thickness of the sample exceeds the depletion width and/or the absorption length, at which point the photocurrent decays exponentially due to carrier diffusion limitations. The dotted blue line depicts a case with nearly infinite recombination at the back surface, e.g. a true ohmic contact. Minority carrier loss at the back contact results in a decrease in the magnitude of the photocurrent relative to the base case, but the shape of the curve is essentially unchanged in the region where the sample thickness exceeds the sum of the depletion width and the absorption length. The grey dot-dashed line depicts the limiting case of eqn (2), in which the minority-carrier diffusion length is shorter than the absorption length. In this limiting case, the photocurrent also decreases exponentially with thickness, but for weakly-absorbed light the parameter that can be extracted from the slope of the curve is only the absorption length, $1 / \alpha$, as opposed to the minority-carrier diffusion length.

The ability to extract quantitative information from the region in which the photocurrent rises $v s$. lateral distance, representing conditions for which the thickness of the sample is comparable to the diffusion length, is limited by various behaviours that are dependent upon the width of the depletion region, the rate of recombination at the back contact, and the absorption length. Hence, the analysis was only performed using data from the thickness range that produced an exponential decay in the photocurrent as a function of the sample thickness. For these calculations, the thickness of the absorber within the illuminated area was assumed to be uniform; however, $L_{\mathrm{p}}$ is still extracted from the slope even when the thickness of the absorber varies within the illuminated area, provided that the gradient change of the thickness within the illuminated area is small compared to the diffusion length (see ESI $\dagger$ ).

The mathematical analysis and graphical examples therefore show that $L_{\mathrm{p}}$ can be obtained by scanning a light beam along the back side of a wedge-shaped absorber provided that: (1) the film is thick enough to reach the exponentially decaying regime, but not so thick that the photocurrent is negligible; (2) the minoritycarrier diffusion length is greater than the absorption length; (3) the film is sufficiently dense that minority-carrier transport is predominantly one-dimensional, implying that any grain boundaries or inhomogeneity along the film thickness are inactive toward carrier collection or recombination. Defects along the film thickness may affect the minority carriers, and the parameter then obtained using this technique is not necessarily the bulk diffusion length (see the ESI $\dagger$ and Discussion section regarding porosity), though the measured effective minoritycarrier collection length is still relevant to device design.

\section{Experimental methods}

To provide a sample for proof-of-concept experiments, a primegrade n-type Si wafer (University Wafer) was polished at $a<1^{\circ}$ angle to produce a wedge, using a tripod polisher and diamond lapping films. After polishing, the sample was immersed for a few $\mathrm{s}$ in $10 \% \mathrm{HF}$ (aq.) before metal deposition. A Pt Schottky contact was then deposited by electron-beam evaporation over most of the flat side of the $\mathrm{Si}$, and $\mathrm{In} / \mathrm{Ga}$ eutectic was used to make ohmic contact to the edge of the bulk wafer $1 \mathrm{~mm}$ away from the closest point of the illuminated area. The Pt/Si junction showed leaky-diode-type behaviour with a quality factor of 3-4. The quality of the diode was however sufficient to allow collection of photogenerated minority carriers at short circuit. The dopant density of the wafer was $5.3 \times 10^{16} \mathrm{~cm}^{-3}$, and the sample had an expected minority-carrier diffusion length ${ }^{17}$ of 100-200 $\mu \mathrm{m}$. After fabrication, the sample was left in air before the photocurrent measurement, so the back (lapped) surface was likely unpassivated and had a high surface recombination velocity.

Tungsten trioxide, $\mathrm{WO}_{3}$, and bismuth vanadate, $\mathrm{BiVO}_{4}$, were chosen as proof-of-concept materials to demonstrate the technique on candidate photoanodes for use in a PEC device. ${ }^{\mathbf{1 8 - 2 0}}$ Films of $\mathrm{WO}_{3}$ were deposited onto substrates using reactive RF magnetron sputtering from a 2 "-diameter tungsten target at 200 W with $20-25 \% \mathrm{O}_{2}$ in Ar at 6-10 mTorr. For optical characterization, microscope slides were used as substrates; for thickness profiles, Si wafer pieces were used as substrates; and for electrochemical measurements, $\mathrm{F}: \mathrm{SnO}_{2}$-coated (FTO) glass pieces (Pilkington TEC15) were used as substrates. The temperature of the substrates during sputtering was varied from $180-400{ }^{\circ} \mathrm{C}$. The targeted maximum film thicknesses ranged from 1.5 to 4 $\mu \mathrm{m}$, and the growth rate was $\sim 500 \mathrm{~nm} \mathrm{~h}^{-1}$. To develop a thickness gradient in the deposited $\mathrm{WO}_{3}$ films, during sputtering a shutter was placed $\sim 1 \mathrm{~cm}$ above one-half of each substrate material. The thickness gradient developed over a length of $\sim 2 \mathrm{~cm}$, was clearly visible, and was characterized via optical reflectance fringes, as detailed in the ESI. $\uparrow \mathrm{BiVO}_{4}$ films were synthesized on FTO glass substrates using a published spin-coating procedure. ${ }^{21} 18$ deposition cycles were used to make the films that were sintered at $500{ }^{\circ} \mathrm{C}$ for 10 minutes after each cycle. $\mathrm{BiVO}_{4}$ films carefully mounted and levelled on a tripod were polished into a wedge with gentle polishing using $50 \mathrm{~nm}$ colloidal diamond slurry (Buehler MetaDi Supreme 0.05 $\mu \mathrm{m}$ ) on a porous polyurethane pad (Eminess Politex Reg) for 20 minutes on a polishing wheel at $100 \mathrm{rpm}$, and the thickness profile was characterized with a series of focused ion beam (FIB) cross-sections. To insulate any electrochemically active pores on porous films, a $10 \mathrm{~nm}$ layer of aluminum oxide was deposited by atomic layer deposition (ALD) (Cambridge Nanotechnology) on $\mathrm{WO}_{3}$ and $\mathrm{BiVO}_{4}$ films. A long diffusion time $(20 \mathrm{~s})$ was used to allow the precursor to fully diffuse inside the pores. To expose the top surface, the samples were gently polished using the same colloidal diamond slurry on the polyurethane pad for 10 minutes at $100 \mathrm{rpm}$. A detailed analysis was performed to realize and verify a conformal and insulating ALD-treatment of the pores and to obtain a uniform polishing of the top surface (see $\mathrm{ESI} \dagger$ ).

Electrochemical measurements were performed in a custom electrochemical cell that was equipped with a fixed-position working electrode, a Pt mesh counter electrode, and a $\mathrm{Ag} / \mathrm{AgCl}$ 
reference electrode (BASi) having an electrode potential of $E(\mathrm{AgCl} / \mathrm{Ag})$. Unstirred, air-saturated reagent-grade $1 \mathrm{M} \mathrm{H}_{2} \mathrm{SO}_{4} /$ $2.4 \mathrm{M} \mathrm{CH}_{3} \mathrm{OH}$ (aq.) was used as the electrolyte for $\mathrm{WO}_{3}$ films and $0.5 \mathrm{M} \mathrm{Na}_{2} \mathrm{SO}_{3} / 0.1 \mathrm{M} \mathrm{K}_{2} \mathrm{HPO}_{4}$ buffer solution ( $\mathrm{pH} \mathrm{7)}$ for $\mathrm{BiVO}_{4}$. Control experiments verified that the stirring and/or electrode placement did not affect the measured data, due to the small currents involved and the high concentration of the electrolyte. A $1 \mathrm{~mm}$ diameter spot illumination scanned along the back side of the wedge-shaped samples, with a modulation frequency of $0.5 \mathrm{~Hz}$ and $20 \mathrm{~Hz}$, was used for $\mathrm{WO}_{3}$ and $\mathrm{BiVO}_{4}$ signals, respectively. The resulting photocurrent was measured with an SRS830 lock-in amplifier connected to a BioLogic SP-200 potentiostat.

A compact, $2 \mathrm{~mW} 365 \mathrm{~nm}$ UV light-emitting diode (LED) (Thorlabs) was selected to balance the requirement for strong absorption by $\mathrm{WO}_{3}$ and transmission through the FTO substrate. A $455 \mathrm{~nm}$ LED and a $500 \mathrm{~nm}$ LED that produced an optical output power similar to that of the $365 \mathrm{~nm}$ LED were also used as the illumination sources for data collection from the Si wedge sample. A Newport $150 \mathrm{~W}$ Xe arc lamp coupled to a Cornerstone $1 / 4 \mathrm{~m}$ monochromator was used for spectrally varied photocurrent measurements of $\mathrm{BiVO}_{4}$.

Because methanol was used as a hole scavenger for $\mathrm{WO}_{3}$, current doubling occurred in this system. The photocurrent was monitored a function of the methanol concentration, and a high concentration of methanol $(2.4 \mathrm{M})$ was chosen to preserve the current doubling at the highest photocurrent value and to achieve a light-limited current throughout the scanned range of sample thicknesses.

Scanning electron micrography was performed using a fieldemission scanning electron microscope (SEM, Sirion, FEI). Xray diffraction data (XRD) were collected on a PANalytical X'Pert Pro MRD operated at $45 \mathrm{kV}$ using $\mathrm{Cu}-\mathrm{K} \alpha$ radiation.

\section{Results}

\section{Silicon wedge}

Fig. 2 shows the short-circuit photocurrents that were measured as the illumination from each of three strongly absorbed wavelengths was scanned along the back side of the Si wedge/Pt Schottky junction. The peak photocurrents for the 365,455 , and $500 \mathrm{~nm}$ illumination sources corresponded to incident photonto-electron conversion efficiencies (IPCE) of $3 \times 10^{-5}, 2 \times 10^{-3}$, and $6 \times 10^{-3}$, respectively. The observed photocurrent profiles were consistent with the analytical one-dimensional model for the case of high rates of recombination at the back surface (blue dotted trace in Fig. 1b). The rate of exponential decrease of the photocurrent with increasing sample thickness was nearly identical for all three wavelengths. The linear fit for each wavelength (shown as dotted lines in Fig. 2) was used to calculate a value of $L_{\mathrm{p}}$, yielding a value of $L_{\mathrm{p}}=168 \pm 4 \mu \mathrm{m}$.

\section{Tungsten oxide wedge}

During deposition of the $\mathrm{WO}_{3}$, variations in the sputtering temperature as well as in the partial pressure of oxygen produced films with a variety of morphologies and

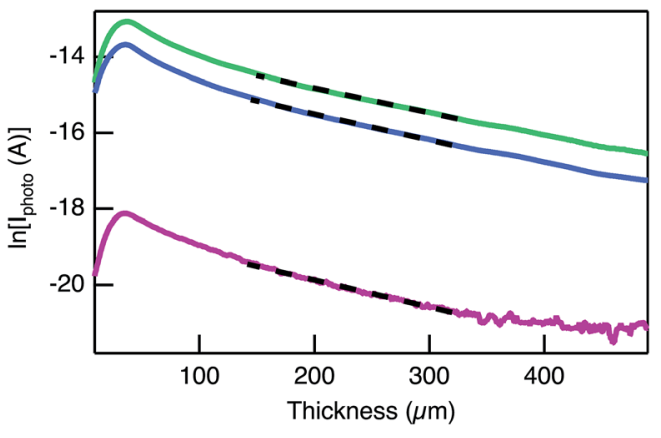

Fig. 2 Photocurrent as a function of thickness for a Schottky diode made using a polished Si wedge. From top to bottom, the illumination wavelengths were 500, 455, and $365 \mathrm{~nm}$, respectively. The dotted lines are the linear fits that were used to determine the diffusion length and produced a value of $L_{p}=168 \mu \mathrm{m}$.

photoelectrochemical responses. ${ }^{\mathbf{1 9 , 2 0}}$ Films produced using relatively low deposition temperatures (down to $200{ }^{\circ} \mathrm{C}$ ) showed more ideal photocurrent $v s$. thickness behaviour than films deposited using higher temperatures (400-500 $\left.{ }^{\circ} \mathrm{C}\right)$. Crosssectional SEM images showed columnar growth at all temperatures as shown in the Fig. 3a inset, but films grown using lower temperatures showed denser packing and smoother top surfaces than those grown at higher temperatures. All of the films were polycrystalline, and displayed XRD patterns consistent with the monoclinic phase of $\mathrm{WO}_{3}$ (Fig. 3a and the $\mathrm{ESI}_{\dagger} \dagger$ ). However, the presence of orthorhombic or sub-stoichiometric
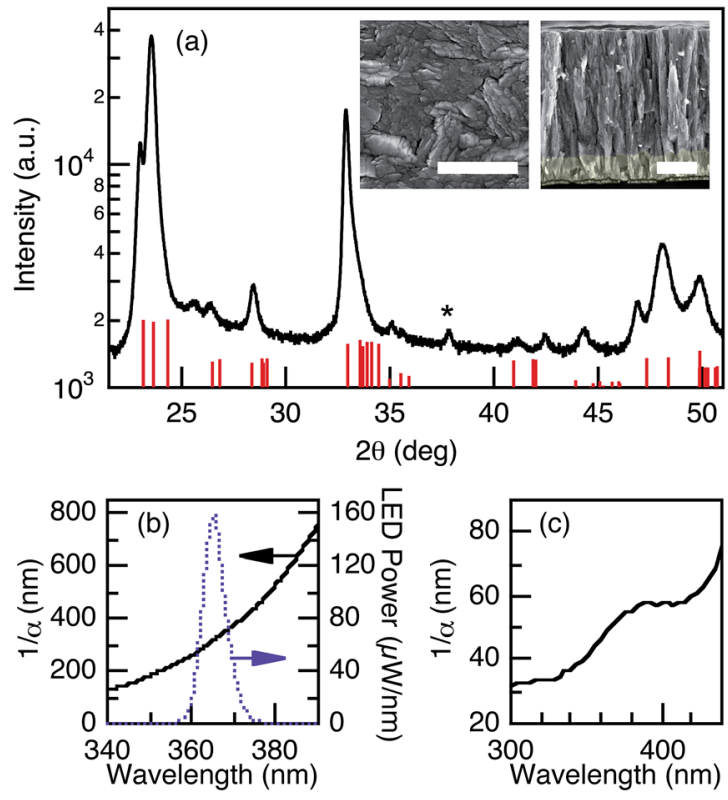

Fig. 3 Photoelectrode film properties. (a) X-ray diffraction data for a $\mathrm{WO}_{3}$ film. The monoclinic powder peaks are shown as red lines at the bottom and the substrate peak is labelled with a star. Insets: top view (left) and cross-section (right) SEM images of a film with a $500 \mathrm{~nm}$ scale bar and with the $\mathrm{F}: \mathrm{SnO}_{2}$ film shaded yellow. (b) Optical absorption length of $\mathrm{WO}_{3}$ and (c) $\mathrm{BiVO}_{4}$ thin films. The purple dotted line shows the UV LED spectrum that determines the minimum measureable diffusion length. 
$\left(\mathrm{WO}_{2.9}\right)$ phases seen in previous work ${ }^{\mathbf{1 9}}$ can not be ruled out, due to the highly oriented film growth and the mutual similarity of the various XRD patterns. The tungsten oxide films displayed typical onset potentials and PEC behaviour (ESI $\dagger$ ).

To verify that the UV illumination was absorbed sufficiently strongly by the $\mathrm{WO}_{3}$, the spectral absorbance of the $\mathrm{WO}_{3}$ films of deposited on a glass substrate was determined from optical transmission and reflection measurements. Fig. $3 \mathrm{~b}$ shows the $\mathrm{WO}_{3}$ absorption length as well as the spectrum used to illuminate the film. The minimum measurable value of $L$ is determined by the absorption length at the illumination wavelength. The UV LED used in the $\mathrm{WO}_{3}$ experiments has a spectral bandwidth centered at $365 \mathrm{~nm}$, and significant intensity extends to $375 \mathrm{~nm}$. Thus the estimated minimum measurable diffusion length was $400 \mathrm{~nm}$ for these sputtered $\mathrm{WO}_{3}$ thin films. Such detailed measurements of the absorption coefficient may not be necessary for materials with smaller band gaps.

The photocurrent was recorded as a function of thickness with the working electrode set to $+1 \mathrm{~V}$ vs. $E(\mathrm{AgCl} / \mathrm{Ag})$. Fig. $4 \mathrm{a}$ shows the exponential decay of the photocurrent with thickness for a compact $\mathrm{WO}_{3}$ film deposited at $200{ }^{\circ} \mathrm{C}$. The slope of the exponential decrease was used to calculate a diffusion length of $700 \pm 40 \mathrm{~nm}$ for this film, with the error estimated based upon taking the slope at different points on the profile. The experimentally obtained value was well above the absorption length, so the measurement was therefore performed within the regime valid for the technique.
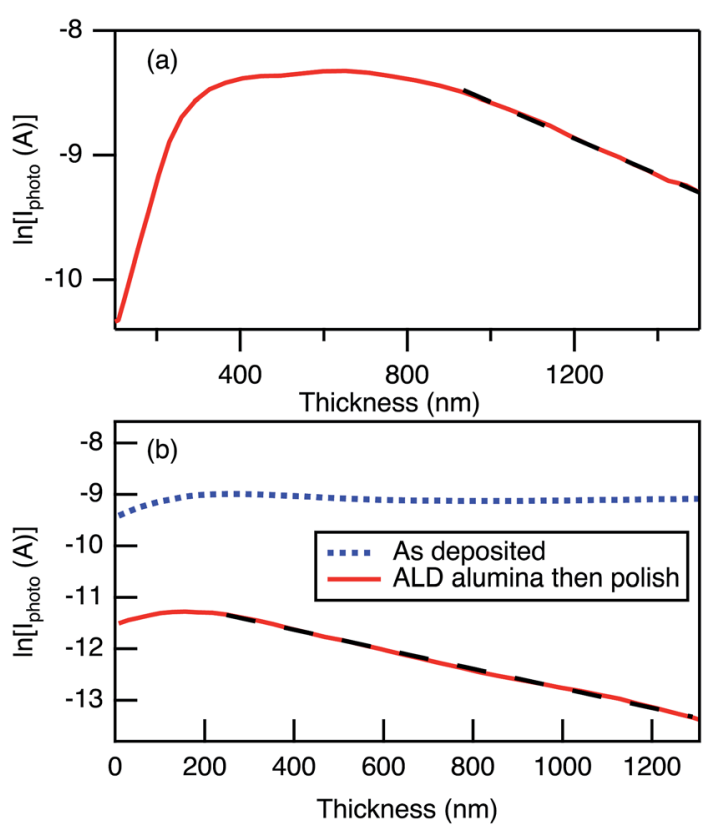

Fig. 4 (a) Photocurrent as a function of thickness for a $\mathrm{WO}_{3}$ wedge sputtered at $200{ }^{\circ} \mathrm{C}$ in contact with a liquid junction. The black dotted line shows the linear fit used to determine the effective minoritycarrier diffusion length, producing a value of $L_{p}=700 \mathrm{~nm}$. (b) $\mathrm{WO}_{3}$ deposited at $400{ }^{\circ} \mathrm{C}$ and subsequently insulated with ALD alumina and polished to expose the top surface. Black dotted line corresponds to a diffusion length of $530 \mathrm{~nm}$.

\section{Porous tungsten oxide wedge}

The wedge technique requires that carrier collection be onedimensional, and the presence of pores changes the apparent film thickness with a liquid contact as discussed in the ESI. $\dagger$ Although low temperature deposition $\left(200{ }^{\circ} \mathrm{C}\right)$ enabled dense and void-free $\mathrm{WO}_{3}$ films, films grown at 300-500 ${ }^{\circ} \mathrm{C}$ were found to be very porous, ${ }^{20}$ and as deposited, the back-illuminated photocurrent did not decrease with increasing film thickness (Fig. 4b). To insulate any electrochemically active pores or grain sidewalls on porous films, we coated the entire sample surface with a $10 \mathrm{~nm}$ layer of ALD aluminum oxide, sufficiently thick to completely fill the pores. The samples were then gently polished to expose the top surface (ESI $\dagger$ ).

Fig. $4 \mathrm{~b}$ shows the exponential decay of the photocurrent recovered after the ALD treatment and polishing for $\mathrm{WO}_{3}$ samples grown at $400{ }^{\circ} \mathrm{C}$. A diffusion length of $530 \mathrm{~nm}$ is obtained which is lower than for the samples deposited at 200 ${ }^{\circ} \mathrm{C}$. The discrepancy could be due to an actual difference in the bulk of the grains, the properties of the grain boundaries, or increased grain-boundary recombination where the aluminum oxide was in contact with the tungsten oxide. For these samples, no dependence on voltage was found over the range 0.6 to $1.2 \mathrm{~V}$ vs. $E(\mathrm{AgCl} / \mathrm{Ag})$.

\section{Bismuth vanadate wedge}

The wedge technique was applied next to find the diffusion length of porous $\mathrm{BiVO}_{4}$ films synthesized by a solution-cast method. The same ALD and polishing procedure was applied on $\mathrm{BiVO}_{4}$ films to insulate the pores after polishing the films into a wedge with thickness variation from $10 \mathrm{~nm}$ to $160 \mathrm{~nm}$. To enable short-wavelength excitations, we used a Xe lamp coupled to a monochromator for photocurrent measurements. Fig. 5 shows the photoresponse vs. thickness at different excitation wavelengths for $\mathrm{BiVO}_{4}$ biased potentiostatically at $+0.6 \mathrm{~V} v s$. $E(\mathrm{AgCl} / \mathrm{Ag})$. As the excitation wavelength increased (from bottom plots towards top) the absorption length increased (Fig. 3c). The diffusion length is given by the slope at short wavelengths (bottom), at which the absorption length is much smaller than the diffusion length. A line fit to data gave a slope of $75 \mathrm{~nm}^{-1}$ at wavelengths ranging from $310 \mathrm{~nm}$ to $330 \mathrm{~nm}$. As the excitation wavelength was further increased from $340 \mathrm{~nm}$ (cyan) to $440 \mathrm{~nm}$ (black), the absorption length was closer to the diffusion length, and the slope changed from $79 \mathrm{~nm}^{-1}$ to 147 $\mathrm{nm}^{-1}$. This dependence on the excitation wavelength is expected based on the film thickness and the absorption length values in Fig. 3c. These results reveal that the wedge technique can retrieve diffusion lengths as small as $75 \mathrm{~nm}$, even for very small film thicknesses, by using a proper excitation wavelength.

\section{Discussion}

The diffusion length for tungsten oxide measured using the wedge technique implemented herein is similar to the range of $L_{\mathrm{p}}=0.5-5 \mu \mathrm{m}$ values reported previously. ${ }^{18,22}$ The variability in $L_{\mathrm{p}}$ between different samples can be ascribed to variations in the material morphology and variations in the density and 


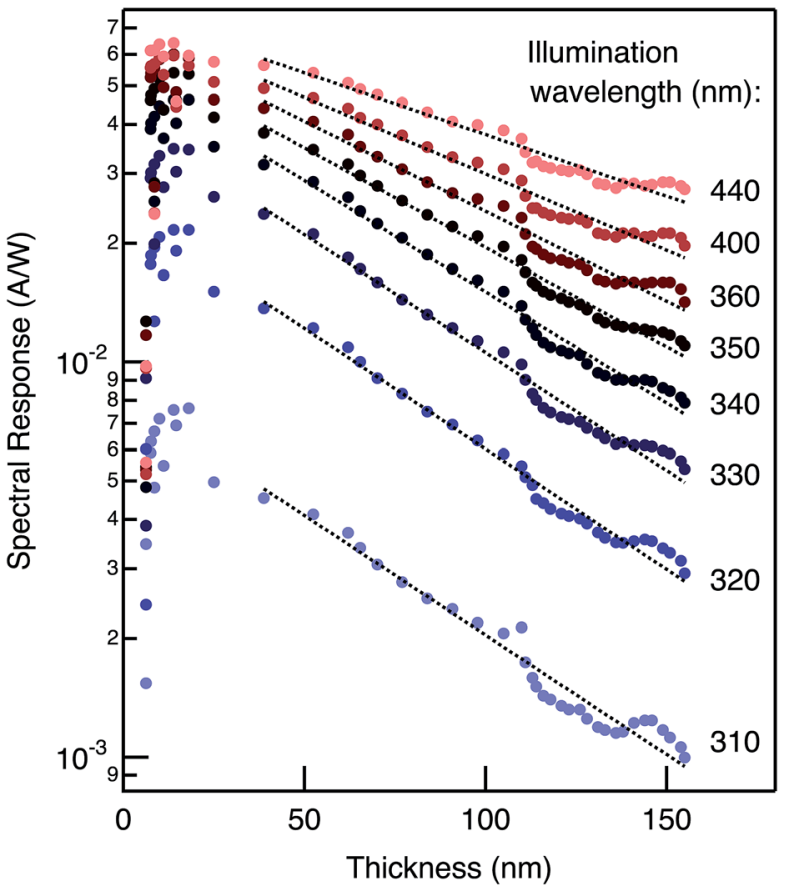

Fig. 5 Photocurrent as a function of thickness for bismuth vanadate at different excitation wavelengths. The slope is equivalent to the diffusion length at short wavelengths at which the absorption length is smaller than the diffusion length. A line fit to data gives a slope of 75 $\mathrm{nm}$ at wavelengths of $300 \mathrm{~nm}$ (blue), 310 (green) and $320 \mathrm{~nm}$ (red). As the excitation wavelength is further increased from $340 \mathrm{~nm}$ (cyan) to $440 \mathrm{~nm}$ (black), the absorption length is closer to the diffusion length, and the slope changes from $79 \mathrm{~nm}$ to $147 \mathrm{~nm}$.

properties of grain boundaries as a result of different sample preparation conditions. The presence of grain boundaries can also affect the minority-carrier lifetime, so in many cases the measured effective diffusion length may not represent the bulk single-crystal diffusion length. However, the measured value nevertheless represents the physical distance that a minority carrier can travel on average in a material of interest.

In principle the measurement technique used herein does not require a wedge, because any well-controlled variation in thickness across a single sample or multiple samples should be sufficient to allow determination of $L_{\mathrm{p}}$. Wedges were however chosen herein to reduce the uncertainty that would result from sample-to-sample variation. The fabrication of a sample with a deliberately varied thickness can require non-traditional processing, but extraction of $L_{\mathrm{p}}$ requires less detailed measurements and fewer assumptions about the material under study compared to the spectral response or EBIC techniques. The traditional spectral response technique with the Gärtner anal$y \operatorname{sis}^{23}$ yields the sum of depletion width and diffusion length, however the technique used herein does not require knowledge of the depletion width. Additionally, for new semiconductor materials, the numerous choices for redox couples and electrolytes make liquid junctions easier to form and test than solidstate junctions.

The illuminated lapped side of the Si wedge had numerous micron-scale scratches that were produced by the polishing procedure. The lower photocurrents obtained using the shorter wavelength illumination sources, and the low IPCE values, show that the surface recombination rate was relatively high for such samples, compared to typical values observed for smooth and passivated Si surfaces. However, the close agreement between the slope of the $\ln \left[J_{\text {photo }}\right] v s$. thickness data for the three different excitation wavelengths indicates that the values obtained for $L_{\mathrm{p}}$ did not depend upon the quality of the back surface. The measurement is also independent of the absorption length provided that the incident light is strongly absorbed.

When using this wedge technique to obtain minority-carrier diffusion lengths, the system should be in a photocurrent saturation regime, but not fully depleted. As in a spectral response measurement, the device should be in a light-limited voltage regime, because non-ideal effects such as junction-side recombination will otherwise produce a violation of the underlying assumptions used herein to analyse the data and extract $L_{\mathrm{p}}$. For the $\mathrm{WO}_{3}$ samples used herein, an applied reverse bias of $+1 \mathrm{~V} v$ s. $E(\mathrm{AgCl} / \mathrm{Ag})$ was sufficient to obtain data that did not depend on voltage.

In a porous material, the electrolyte penetration into the pores can allow carrier collection below the surface and can alter the electric field distribution and depletion region geometry, thus violating the assumptions of the simple 1-D theory developed above. However, by insulating the pores with ALD $\mathrm{Al}_{2} \mathrm{O}_{3}$ to prevent electrolyte contact and electron transfer to the active material in the pores, the junction was confined to the top polished surface. Despite the insulation, electrolyte penetration into the pores can alter the field distribution in the thin film due to capacitive charging, if the depletion width of the semiconductor material at the specified applied bias is comparable to, or smaller than, the average separation between two adjacent pores. This issue can be circumvented by filling the pores with an ALD film having a thickness that is equal to or larger than the pore radius. The method of insulating porosity and sidewalls by ALD and polishing to achieve 1D carrier transport in photoelectrochemical cells facilitates the use of this technique on a wide variety of materials. Future work will extend the technique to other electrode materials such as those deposited using methods such as spray pyrolysis or electrodeposition with subsequent polishing to form a thickness gradient. A technique that allows rapid measurement of the minority-carrier diffusion length will assist in screening new semiconducting materials that are candidates for use in PEC devices.

\section{Conclusions}

The back-illuminated wedge technique can provide a measurement of the minority-carrier diffusion length for semiconducting materials even without detailed knowledge of absorption or electrical properties of the sample of interest. The calculations of diffusive transport in a quasi-neutral semiconductor and collection at a liquid-induced junction show that the measurement is largely insensitive to the depletion width and recombination at the back surface. The utility of the technique was demonstrated using a polished Si wedge, a sputtered $\mathrm{WO}_{3}$ wedge, and polished $\mathrm{BiVO}_{4}$ wedge, and the diffusion 
lengths that were obtained ranged from $75 \mathrm{~nm}$ to $170 \mu \mathrm{m}$ in agreement with the expected values. The wedge geometry is best applied to a dense material rather than a nanostructured electrode, but the measurement in turn guides the nanostructuring design. The light absorption length sets the lower limit of the diffusion lengths that can be measured with this technique, and as the band gap decreases or becomes direct, the lower limit of measurable diffusion lengths also decreases.

\section{Acknowledgements}

This material is based upon work performed by the Joint Center for Artificial Photosynthesis, a DOE Energy Innovation Hub, supported through the Office of Science of the U.S. Department of Energy under Award Number DE-SC0004993. We thank C. Garland for advice on polishing techniques.

\section{References}

1 N. S. Lewis and D. G. Nocera, Proc. Natl. Acad. Sci. U. S. A., 2006, 103, 15729-15735.

2 M. G. Walter, E. L. Warren, J. R. McKone, S. W. Boettcher, Q. Mi, E. A. Santori and N. S. Lewis, Chem. Rev., 2010, 110, 6446-6473.

3 T. F. Jaramillo, S.-H. Baeck, A. Kleiman-Shwarsctein, K.-S. Choi, G. D. Stucky and E. W. McFarland, J. Comb. Chem., 2004, 7, 264-271.

4 C. J. Taylor and S. Semancik, Chem. Mater., 2002, 14, 16711677.

5 R. Potyrailo, K. Rajan, K. Stoewe, I. Takeuchi, B. Chisholm and H. Lam, ACS Comb. Sci., 2011, 13, 579-633.

6 Z. Li, W. Luo, M. Zhang, J. Feng and Z. Zou, Energy Environ. Sci., 2013, 6, 347-370.

7 S. Mao, Appl. Phys. A: Mater. Sci. Process., 2011, 105, 283-288.
8 Z. Chen, T. F. Jaramillo, T. G. Deutsch, A. KleimanShwarsctein, A. J. Forman, N. Gaillard, R. Garland, K. Takanabe, C. Heske, M. Sunkara, E. W. McFarland, K. Domen, E. L. Miller, J. A. Turner and H. N. Dinh, J. Mater. Res., 2010, 25, 3-16.

9 H. J. Leamy, J. Appl. Phys., 1982, 53, R51-R80.

$10 \mathrm{~J}$. W. Orton and P. Blood, The Electrical Characterization of Semiconductors: Measurement of Minority Carrier Properties, Academic Press, London, 1990, pp. 217-278.

11 W. H. Hackett Jr, R. H. Saul, R. W. Dixon and G. W. Kammlott, J. Appl. Phys., 1972, 43, 2857-2868.

12 C. J. Wu and D. B. Wittry, J. Appl. Phys., 1978, 49, 2827-2836. 13 A. Boudjani, G. Bassou, T. Benbakhti, M. Beghdad and B. Belmekki, Solid-State Electron., 1995, 38, 471-475.

14 L. Kronik and Y. Shapira, Surf. Sci. Rep., 1999, 37, 1-206.

15 J. Navas, E. Guillén, R. Alcántara, C. Fernández-Lorenzo, J. Martín-Calleja, G. Oskam, J. Idígoras, T. Berger and J. A. Anta, J. Phys. Chem. Lett., 2011, 2, 1045-1050.

16 T. Lana-Villarreal, D. Monllor-Satoca, R. Gómez and P. Salvador, Electrochem. Commun., 2006, 8, 1784-1790.

17 J. A. del Alamo and R. M. Swanson, Solid State Electron., 1987, 30, 1127-1136.

18 M. A. Butler, J. Appl. Phys., 1977, 48, 1914-1920.

19 B. Marsen, B. Cole and E. L. Miller, Sol. Energy Mater. Sol. Cells, 2007, 91, 1954-1958.

20 V. S. Vidyarthi, M. Hofmann, A. Savan, K. Sliozberg, D. König, R. Beranek, W. Schuhmann and A. Ludwig, Int. J. Hydrogen Energy, 2011, 36, 4724-4731.

21 T. H. Jeon, W. Choi and H. Park, Phys. Chem. Chem. Phys., 2011, 13, 21392-21401.

22 H. Wang, T. R. Lindgren, J. He, A. Hagfeldt and S.-E. Lindquist, J. Phys. Chem. B, 2000, 104, 5686-5696.

23 W. W. Gärtner, Phys. Rev., 1959, 116, 84-87. 